\title{
Bacillus bacteria in the resistance of potato plants to viruses
}

Burkhanova G.F.*, Sorokan A.V., Sarvarova E.R., Iskandarova Z.M., Maksimov I.V. Institute of Biochemistry and Genetics, UFRC RAS, Ufa, Russia

*e-mail:guzel_mur@mail.ru

Plant viruses have a significant negative impact on major cultures of agronomic importance provoking a wide range of symptoms, representing a serious threat to global food security. Subsistence crops are often infected with viruses that cannot be controlled with pesticides. Biopreparations based on endophytic microorganisms may be an alternative. There are genes of ribonucleases (RNases) produced by many species of Bacillus. The synthesis of secreted degradation enzymes including RNases, this is one way to adapt bacteria to variable habitat conditions. The secreted RNase allows bacilli to have a broad spectrum of biological activities. In low concentrations, they stimulate the growth and physiological functions of plants and microorganisms. In high concentrations, they have antiviral activity. The microbial RNases are potential therapeutic agents, thus the assessment of plant protection against viruses by endophytic bacteria is becoming an important practical problem. In our laboratory there is a collection of bacteria produced various metabolites and have RNase activity. We compared growth and development indicators of potato treated with $B$. subtillis $26 \mathrm{D}$ and $B$. thuringiensis. The results showed that the inoculation increased the grain yield by $0.2-0.7 \mathrm{t} \cdot \mathrm{ha}^{-1}$. In addition, the treatment of potato plants with bacteria of Bacillus significantly reduced the infection of potato plants with viruses. The prevalence of the disease in potato plants was significantly reduced from $75 \%$ in the control to $18 \%$ (B. subtillis $26 \mathrm{D})$ and $33 \%$ (B. thuringiensis) in the inoculated plants. Similarly, the index of viral infection development decreased from $5-10 \%$ in the control to $1-5 \%$ in the inoculated plants. The further study of molecular mechanisms related to bacterial induction of plant defense reactions in response to viral infections will lead to a better understanding of stress resistance problems. The endophytic microorganisms studied in this report may become basis for creation of biological agents for plant protection.

Acknowledgements: This study was supported by the Russian Science Foundation (project No. 19-46-02004). 